\title{
Multi-site reproducibility of prefrontal-hippocampal connectivity estimates by stochastic DCM
}

\author{
D. Bernal-Casas ${ }^{\text {a,b,* }}$, E. Balaguer-Ballester ${ }^{\mathrm{a}, \mathrm{c}}$, M.F. Gerchen ${ }^{\mathrm{a}, \mathrm{b}}$, S. Iglesias ${ }^{\mathrm{d}}$, H. Walter ${ }^{\mathrm{e}}$, A. Heinz ${ }^{\mathrm{e}}$, \\ A. Meyer-Lindenberg ${ }^{\text {a,f }}$, K.E. Stephan ${ }^{\text {d,g,h }}$, P. Kirsch ${ }^{\text {a,b }}$ \\ a Bernstein-Center for Computational Neuroscience Heidelberg-Mannheim, Central Institute of Mental Health, University of Heidelberg, Mannheim, Germany \\ ${ }^{\mathrm{b}}$ Department of Clinical Psychology, Central Institute of Mental Health, University of Heidelberg, Mannheim, Germany \\ c School of Design, Engineering and Computing, Bournemouth University, UK \\ d Translational Neuromodeling Unit (TNU), Institute for Biomedical Engineering, University of Zurich and ETH Zurich, Zurich, Switzerland \\ e Department of Psychiatry and Psychotherapy, Charité Universitätsmedizin, Berlin, Germany \\ ${ }^{\mathrm{f}}$ Department of Psychiatry and Psychotherapy, Central Institute of Mental Health, University of Heidelberg, Mannheim, Germany \\ ${ }^{g}$ Wellcome Trust Centre for Neuroimaging, University College London, London, UK \\ ${ }^{\mathrm{h}}$ Laboratory for Social and Neural Systems Research (SNS), University of Zurich, Switzerland
}

\section{A R T I C L E I N F O}

\section{Article history:}

Accepted 29 May 2013

Available online 5 June 2013

\section{Keywords:}

fMRI

Working memory

Effective connectivity

SDCM

PFC

$\mathrm{HF}$

Schizophrenia

\begin{abstract}
A B S T R A C T
This study examined the reproducibility of prefrontal-hippocampal connectivity estimates obtained by stochastic dynamic causal modeling (sDCM). 180 healthy subjects were measured by functional magnetic resonance imaging (fMRI) during a standard working memory N-Back task at three different sites (Mannheim, Bonn, Berlin; each with 60 participants). The reproducibility of regional activations in key regions for working memory (dorsolateral prefrontal cortex, DLPFC; hippocampal formation, HF) was evaluated using conjunction analyses across locations. These analyses showed consistent activation of right DLPFC and deactivation of left HF across all three different sites. The effective connectivity between DLPFC and HF was analyzed using a simple two-region SDCM. For each subject, we evaluated sixty-seven alternative sDCMs and compared their relative plausibility using Bayesian model selection (BMS). Across all locations, BMS consistently revealed the same winning model, with the 2-Back working memory condition as driving input to both DLPFC and HF and with a connection from DLPFC to HF. Statistical tests on the SDCM parameter estimates did not show any significant differences across the three sites. The consistency of both the BMS results and model parameter estimates indicates the reliability of sDCM in our paradigm. This provides a basis for future genetic and clinical studies using this approach.
\end{abstract}

(c) 2013 Elsevier Inc. All rights reserved.

\section{Introduction}

Estimates of functional and effective brain connectivity are essential for accounts of human brain function. Particularly, when trying to understand brain pathology in major psychiatric disorders like schizophrenia, the connectivity between brain regions (Fornito et al., 2012; Friston and Frith, 1995; Stephan et al., 2009a) plays an important role. However, to describe brain connectivity and its disturbances in psychiatric conditions, reliable methods have to be developed and evaluated that provide robust and replicable estimates of these processes.

In this study, we investigate the applicability and robustness of stochastic dynamic causal modeling (sDCM; Daunizeau et al., 2012; Friston et al., 2011; Li et al., 2011) to investigate effective connectivity between the dorsolateral prefrontal cortex (DLPFC) and the hippocampal formation (HF) during working memory (WM). These regions play an

\footnotetext{
* Corresponding author at: Bernstein-Center for Computational Neuroscience HeidelbergMannheim, Department of Clinical Psychology, Central Institute of Mental Health (CIMH), J 5 , 68159 Mannheim, Germany. Fax: + 4962117036505.

E-mail address: bernalgps@gmail.com (D. Bernal-Casas).
}

important role in pathophysiological theories of schizophrenia (Barch, 2005; Fletcher, 1998; Weinberger et al., 1992), and their connectivity has been studied in numerous studies on the disorder. Impaired PFC$\mathrm{HF}$ connectivity has been described in mouse models of schizophrenia (Sigurdsson et al., 2010), in healthy individuals carrying a genome wide significant gene variant for the disorder (Esslinger et al., 2009; Paulus et al., 2013; Rasetti et al., 2011) as well as in individuals with increased psychosis risk and in first episode patients (Benetti et al., 2009; Crossley et al., 2009). In schizophrenia patients, altered PFC-HF coupling was found under both resting conditions (Zhou et al., 2007, 2008) and during WM tasks (Crossley et al., 2009; Meyer-Lindenberg et al., 2005; Wolf et al., 2009). In fact, the disconnection hypothesis was motivated by a very similar finding of abnormal dorsal lateral prefrontal cortical connectivity with posterior temporal activity during a verbal fluency paradigm which has a significant WM component (Friston et al., 1992).

WM concerns the maintenance and on-line manipulation of information; and is an essential component of executive control for guiding behavior. WM processes temporary store contents; which are continually updated, scanned and manipulated in response to immediate 
processing demands (Baddeley, 1992). WM deficits in schizophrenia have been consistently described (Forbes et al., 2009; Lee and Park, 2005), they are known to be resilient to the treatment and thus may underlie many of the cognitive deficits and symptoms in the disorder (Silver et al., 2003). These deficits cause longer reaction time and less accurate performance in schizophrenic subjects, especially when memory load increases (Goldman-Rakic, 1994; He et al., 2012; Manoach et al., 1999; Park and Holzman, 1992).

Given this background of the importance of WM for theories of schizophrenia, indices of prefrontal-hippocampal coupling during WM could provide markers of cognitive state that may have diagnostic and predictive utility. In a first step, it is important, however, to examine the robustness and reliability of any such measure of connectivity in healthy volunteers. In fact, even without the clinical motivation, examining the reliability of effective connectivity estimates during WM is, in itself, a valuable goal. WM has been studied extensively in human volunteers using fMRI (Callicott et al., 1999; Gazzaley et al., 2004), including several studies of effective connectivity (Deserno et al., 2012; Schlosser et al., 2003). However, to our knowledge, none of these previous studies had the opportunity to conduct a multi-center comparison across different sites and examine the reliability of effective connectivity estimates during WM in depth.

Here, we examined the reproducibility of prefrontal-hippocampal connectivity estimates across 180 healthy subjects, measured under the same paradigm but at three different sites (Mannheim, Bonn, Berlin; each with 60 participants). We used functional magnetic resonance imaging (fMRI) and a well-established task of WM, the N-Back task. The reproducibility of regional activations was evaluated using conjunction analyses. These analyses showed consistent activation of right DLPFC and deactivation of left HF across the three different sites, in accordance with previous studies of the N-back task (Crossley et al., 2009; Meyer-Lindenberg et al., 2005; Wolf et al., 2009). The effective connectivity between DLPFC and HF was then analyzed using a simple two region model whose parameters were estimated using stochastic dynamic causal modeling (sDCM; Daunizeau et al., 2012; Friston et al., 2011; Li et al., 2011). DCM is a framework for formulating physiologically interpretable and generative models of brain responses, such as measured with fMRI (Friston et al., 2003). It rests on Bayesian inversion and comparison of state space models formulated in terms of differential equations (Stephan et al., 2010).

Importantly, stochastic dynamic causal models (sDCMs) allow for uncertainty about both the states and parameters of the model. Previous DCM studies of neuroimaging time series have been limited largely to deterministic DCMs, where uncertainty about the states is ignored (Friston et al., 2003). These are based on ordinary differential equations and assume that there are no random variations in the hidden neuronal and physiological states that mediate the effects of known experimental inputs on observed fMRI responses. This may be a limitation in some instances, because several studies suggest that physiological noise due to stochastic fluctuations in neuronal and vascular responses may need to be taken into account (Biswal et al., 1995; Kruger and Glover, 2001; Riera et al., 2004). As a consequence, there has been growing interest in estimating both the parameters and hidden states of DCMs based upon stochastic differential equations with state noise. Examples of different inversion schemes have been in the DCM literature for a while (Daunizeau et al., 2009; Friston et al., 2008), including a "generalized filtering" scheme (Friston et al., in press).

In our work, we used SDCM based on generalized filtering to estimate the effective connectivity between right DLPFC and left HF and to examine, in a multi-center study, the robustness of these estimates across three different sites (Mannheim, Bonn, Berlin). For each subject, we inverted (fitted) a set of sixty-seven alternative sDCMs and compared their relative plausibility using Bayesian Model Selection (BMS; Penny et al., 2004a; Stephan et al., 2009b). Statistical analyses were employed to test for potential differences across the three sites, both with regard to optimal model structure and concerning the posterior parameter estimates. The consistency of our BMS results and parameter estimates across the three sites indicates the reliability of SDCM in our particular paradigm.

\section{Methods}

\section{Summary of dynamic causal modeling (DCM)}

DCM models the brain as a dynamic system of interconnected regions; while, an experiment is defined as a designed perturbation of the system's dynamics (Friston et al., 2003; Stephan et al., 2010). Any given DCM represents a particular mechanistic model for explaining experimentally obtained measures of brain activity. Even though the mathematical formulation of DCMs differs significantly across different techniques, common neural mechanisms modeled by all DCMs include synaptic connection strengths and their experimental modulation. In contrast to purely statistical models of effective connectivity which characterize inter-regional connectivity in a phenomenological fashion, DCMs strive for neurobiological interpretability of their parameters and this is a core feature which distinguishes them from alternative approaches.

DCMs for fMRI responses are hierarchical models, consisting of two layers. The first layer is a neuronal model that describes the dynamics of interacting neuronal populations in the context of experimental inputs. The second layer is a hemodynamic forward model that characterizes how a given neuronal state translates into observed fMRI responses and serves to account for variations in neurovascular coupling across brain regions and individual subjects. Experimental inputs $u(t)$ enter the model in two different ways: they can elicit responses through direct influences on specific regions (driving inputs), or they can modulate the strength of coupling among regions (modulatory inputs).

Assuming, for simplicity, a single input $u(t)$, the state and measurement equations of a conventional deterministic DCM for fMRI are:

Neuronal state model : $\frac{d x(t)}{d t}=f\left(x(t), \theta_{n}, u(t)\right)=(A+B u(t)) x(t)+C u(t)$

Hemodynamic forward model : $y(t)=g\left(\theta_{h}\right) * x(t)+\varepsilon(t)$

where $x(t)$ represents the neuronal state, $\theta_{n}=(A, B, C)$ are the neuronal parameters, $A$ is a matrix of endogenous connection strengths, $B$ is a matrix of modulatory input strengths, $C$ denotes the strength of direct (driving) inputs, $g\left(\theta_{h}\right)$ is a nonlinear convolution operator that links the neuronal state $x(t)$ to a predicted BOLD signal $y(t)$ via changes in vasodilatation, blood flow, blood volume, and deoxyhemoglobin content (Stephan et al., 2007), $\theta_{h}$ are the hemodynamic parameters, and $\varepsilon(t)$ denotes Gaussian measurement error.

Critically, the neuronal parameters $\theta_{n}$ have some degree of neurobiological interpretability, representing, for instance, synaptic weights and their context-specific modulation. The hemodynamic parameters $\theta_{h}$ are not of scientific research interest because they exhibit strong interdependencies and thus high posterior covariances and low precision, which make it difficult to determine the distinct contribution provided by each parameter.

To summarize, DCM for fMRI responses provides a generative model for explaining measured BOLD time series as the outcome of hidden dynamics in an interconnected network of neuronal populations and its experimentally induced perturbations. Inverting such a model refers to estimate the posterior distribution of the parameters of both the neuronal and the hemodynamic model from observed fMRI responses of an individual subject. 
Stochastic dynamic causal modeling (sDCM)

Stochastic DCMs differ from conventional deterministic DCMs by allowing for endogenous or random fluctuations in unobserved (hidden) neuronal and physiological states, i.e., system or state-noise. Practically, this means that the states are free to fluctuate, in addition to the parameter estimates, to model spontaneous and experimentally induced responses. The mathematical form we use here corresponds to that introduced in Li et al. (2011) which extend Eqs. (1) and (2) by including fluctuation terms for both states and causes:

Neuronal state model : $\begin{aligned} \frac{d x(t)}{d t} & =f\left(x(t), \theta_{n}, v(t)\right)+\omega^{(x)} \\ & =(A+B v(t)) x(t)+C v(t)+\omega^{(x)}\end{aligned}$

Hidden causes model : $\quad v(t)=u(t)+\omega^{(v)}$

Hemodynamic forward model : $\quad y(t)=g\left(\theta_{h}\right) * x(t)+\varepsilon(t)$

The variables have the same meaning as in Eqs. (1) and (2) above. Additionally, $v(t)$ represents the hidden causes, $\omega^{(x)}$ are random neuronal fluctuations, and $\omega^{(v)}$ are random fluctuations in experimental inputs. Importantly, both types of stochastic innovations are assumed to be non-Markovian but show a degree of temporal smoothness; this is a typical feature of biological systems (Friston et al., 2011). In other words, the random state fluctuations $\omega^{(x)}$ are characterized by two hyperparameters $\pi, \sigma$ which describe their precision (inverse variance) and smoothness, given a particular temporal autocorrelation function $V$; for details, see $\mathrm{Li}$ et al. (2011).

The same sort of parameterization applies to the random fluctuations in the inputs $\omega^{(v)}$. States, parameters and hyperparameters from Eqs. (3), (4), and (5) are inferred using generalized filtering, a triple-estimation scheme that employs variational Bayesian techniques (Friston et al., in press). Notably, the presence of stochastic innovations makes sDCMs applicable to both task-driven and "resting-state" fMRI data.

Bayesian model selection (BMS)

In this paper, we used BMS to compare sDCMs that are applied to empirical fMRI data (Penny et al., 2004a; Stephan et al., 2009b). Deciding between several competing models cannot just consider the relative fit to the data but also needs to take into account differences in model complexity; i.e., the number of free parameters and the functional form of the generative model which, for example, determines parameter interdependencies (Pitt and Myung, 2002). Penalizing for model complexity is important: as complexity increases, so does model fit (monotonically). At some point, however, the model will start fitting noise that is specific to a particular measurement (i.e., "over-fitting"). In other words, overly complex models are less generalizable across multiple realizations of the same underlying generative process. Therefore, assuming that all models are equally likely a priori, searching for an optimal model (given a set of alternatives) corresponds to searching the model that represents the best balance between fit and complexity. This is the model that maximizes the model evidence or marginal likelihood which integrates out uncertainty about hidden parameters and states:

$p(y \mid m)=\int p(y \mid \theta, m) p(\theta, m) d \theta$

In this study, we use an estimate of the negative free-energy as an approximation to the log model evidence (Friston et al., 2007) and employ a random effects BMS scheme at the group level that accounts for potential heterogeneity across subjects (Penny et al., 2010; Stephan et al., 2009b).

\section{fMRI data-sets}

\section{Participants}

A total of 180 healthy German participants from three different locations: Mannheim (60), Bonn (60), and Berlin (60), have been analyzed. All participants gave written informed consent, and the study had ethics committee approval by the Universities of Heidelberg, Bonn, and Berlin. Table 1 shows the demographics and behavioral data of participants. We tested for potential systematic differences between subjects examined at the three different locations using a one-way ANOVA or a Kruskal-Wallis test depending on the distribution of the SDCM parameter estimates previously assessed by a Lilliefors test. Regarding age, gender, and education (years of study), no significant differences were found. Concerning the behavioral data, no significant differences in performance and reaction times were found either.

\section{WM paradigm: $N$-Back task}

We utilized the N-Back task (Owen et al., 2005), which requires the temporal tagging and updating of information on each trial, and therefore has a steep difficulty slope with increasing demand (i.e., from 0 -Back to 2-Back).

Our N-Back paradigm used a block design with two conditions: 0 -Back condition and 2-Back condition. In the 0-Back condition (a baseline condition requiring no $\mathrm{WM}$ ), subjects were asked to press the button of the number that they were seeing. In the 2-Back condition, subjects were asked to press the button of the number that they saw two trials before. The study comprised a sequence of 0-Back blocks alternating with 2-Back blocks. Subjects performed four 0-Back blocks and four 2-Back blocks with 15 trials per block.

\section{fMRI data acquisition and preprocessing}

At all three sites, fMRI data were acquired at $3 \mathrm{~T}$ using a Trio TIM (Siemens, Erlangen) whole-body MRI system. 128 contiguous multislice images were obtained with a gradient echo-planar sequence (orientation $=\mathrm{AC}-\mathrm{PC}$ line, number of slices $=28$; slice thickness $=$ $4 \mathrm{~mm}$; slice gap = $1 \mathrm{~mm}$; FOV $=192 \mathrm{~mm} ; \mathrm{TE}=30 \mathrm{~ms} ; \mathrm{TR}=2.00 \mathrm{~s}$; flip angle $=90^{\circ}$; matrix size $=64 \times 64 \times 28$; voxel size $=3.0 \times$ $3.0 \times 5.0 \mathrm{~mm}^{3}$ ).

The fMRI data were analyzed using procedures implemented in Statistical Parametric Mapping (SPM8, Welcome Trust Centre for Neuroimaging, London, UK; http://www.fil.ion.ucl.ac.uk/spm). All

Table 1

Demographics and behavioral data of participants for each location.

\begin{tabular}{|c|c|c|c|c|}
\hline & Mannheim & Bonn & Berlin & P-Value \\
\hline \multirow[t]{2}{*}{ Age [years] } & $33.95 \pm 9.64$ & $31.47 \pm 9.60$ & $34.88 \pm 8.72$ & \multirow[t]{2}{*}{$\mathrm{p}=0.14^{\mathrm{a}}$} \\
\hline & Range 19-49 & Range $18-50$ & Range $18-50$ & \\
\hline Sex [male/female] & $27 / 33$ & $29 / 31$ & $29 / 31$ & $\mathrm{p}=0.92^{\mathrm{a}}$ \\
\hline School education [years] & $15.40 \pm 2.43$ & $15.48 \pm 2.93$ & $15.57 \pm 2.57$ & $\mathrm{p}=0.90^{\mathrm{a}}$ \\
\hline Performance on the 2-Back [\%] & $81.42 \pm 17.22$ & $77.67 \pm 21.28$ & $74.58 \pm 19.45$ & $\mathrm{p}=0.14^{\mathrm{a}}$ \\
\hline Reaction time on the 2-Back [ms] & $472.02 \pm 262.93$ & $461.66 \pm 297.70$ & $577.08 \pm 350.21$ & $\mathrm{p}=0.13^{\mathrm{a}}$ \\
\hline
\end{tabular}

\footnotetext{
a Kruskal-Wallis test.
} 
functional images and the structural image of each subject were preprocessed prior to the statistical analyses. We conducted rigid body motion correction of the functional time-series and "unified segmentation" (Ashburner and Friston, 2005) of the structural image. After co-registration of the realigned functional images to the subject specific structural image, the images were normalized to MNI space using the warping parameters obtained from the unified segmentation procedure applied to the structural image. Finally the functional images were smoothed by applying a $6 \mathrm{~mm}$ full-width at half maximum Gaussian kernel.

\section{fMRI analyses using the general linear model (GLM)}

Following preprocessing, we specified a voxel-wise general linear model (GLM) with two conditions of interest for each participant (0-Back and 2-Back). Each regressor was convolved with a canonical hemodynamic function and its temporal and dispersion derivatives. Contrasts of interest were defined as [2-Back-0-Back] for identifying WM activations in the right DLPFC and [0-Back-2-Back] contrast for identifying deactivations of the left HF.

At the group level, the first-level contrast images were entered into a full factorial design. To identify regions that were consistently (de)activated in participants from all three sites, we used a conjunction analysis (i.e., a "logical AND" analysis based on the conjunction null hypothesis; Nichols et al., 2005). Given our a priori hypothesis, based on the rich literature of N-back fMRI studies (see above), of activation in the right DLPFC and deactivation of left HF during 2-Back vs. 0-Back, we restricted this analysis to our regions of interest which were anatomically defined using the PickAtlas software (WFU PickAtlas, ANSIR Laboratory, Winston-Salem, NC, USA; http://fmri.wfubmc.edu/software/PickAtlas). Significant results were corrected for multiple comparisons using family wise error correction based on Gaussian random field theory.

The significant conjunction results within these anatomical masks were used to specify functionally defined region-of-interest masks for subsequent time series extraction in each subject. Specifically, time series were extracted for each subject by computing the first eigenvariate across all voxels within $6 \mathrm{~mm}$ radius from his/her maximum within the functionally defined ROIs from the group level.

\section{$s D C M$}

The time series extracted from individual maxima within the ROIs defined at the group level were used to fit a set of sixty-seven alternative sDCMs per subject. In relation to the experimental input (cognitive set associated with working memory) we considered two classes of model. In the first set (models 1-7), experimental inputs exerted a driving effect on one or both regions. In the second set (models 8-22) the experimental input changed extrinsic or intrinsic connectivity-such that observed memory related responses were modeled by an interaction between hidden neuronal activity and the connection strengths that shape this activity. Finally, in the third set (23-67), experimental inputs exerted a driving effect on one or both regions and also changed intrinsic connectivity. The structure of these sixty-seven sDCMs is shown by Fig. 1 .

In models 1-7, we systematically examine all combinations of directed connections between DLPFC and HF (from DLPFC to HF, from HF to DLPFC, or both) and where driving inputs enter (in DLPFC, HF, or both). In this set of models, we did not consider models without any driving inputs or without any inter-regional connections. Driving inputs encode the influence of task on the DLPFC-HF network (set to 1 during 2-Back and 0 during 0 -Back). Together with the stochastic innovations whose precision and temporal smoothness are estimated separately for each area (see above), the driving inputs represent and absorb influences from unknown (hidden) regions (Daunizeau et al., 2012; Friston et al., 2011; Li et al., 2011) that change their inputs to the DLPFC-HF network depending on WM load. In other words, in these models, we are not modeling WM load dependent changes in DLPFC-HF connectivity by (bilinear) modulation of activity induced by any condition (i.e., a driving input representing any trial), as is often the case in DCM, but via changes in input to the network. The reason for this choice is that in our experimental design 0-Back and 2-Back blocks are continuously following each other, which means that a driving input representing all task conditions would simply correspond to a constant.

In models 8-22, we consider another type of mechanism. These additional models do not consider the 2-Back WM condition as driving input to HF or DLPFC, instead these regions are purely driven by the stochastic innovations and the 2-Back WM condition modulates all possible combinations of inter-regional and self-connections. In other words, DLPFC and HF are not directly affected by changes in working memory load (in terms of load-dependent driving inputs as in models 1-7 above), but we are modeling WM dependent changes in DLPFC and HF activity through (bilinear) modulation of their connectivity by the 2-Back condition.

In models 23-67, we examine a third set of models. These alternative models consider the 2-Back WM condition as driving input to DLPFC, HF, or both, and modulatory input to all possible combinations of inter-regional and self-connections. In other words, we assume that the 2-Back WM condition elicits responses through direct influences on the regions of interest and modulates the strength of coupling within and between these regions.

For the analyses presented in this paper, we used stochastic DCM for fMRI as implemented in DCM10 in the software package SPM12 (alpha version), code release 4579.

\section{Results}

SPM

The conjunction maps depicted in Figs. 2 and 3 show the common (de)activation pattern across three locations in our anatomically constrained regions of interest. These conjunction analyses showed a consistent activation in the right DLPFC $(x, y, z=45,41,20 ; \mathrm{T}=$ 8.94; $\mathrm{p}=0.05$, FWE-corrected) and consistent deactivation in the left

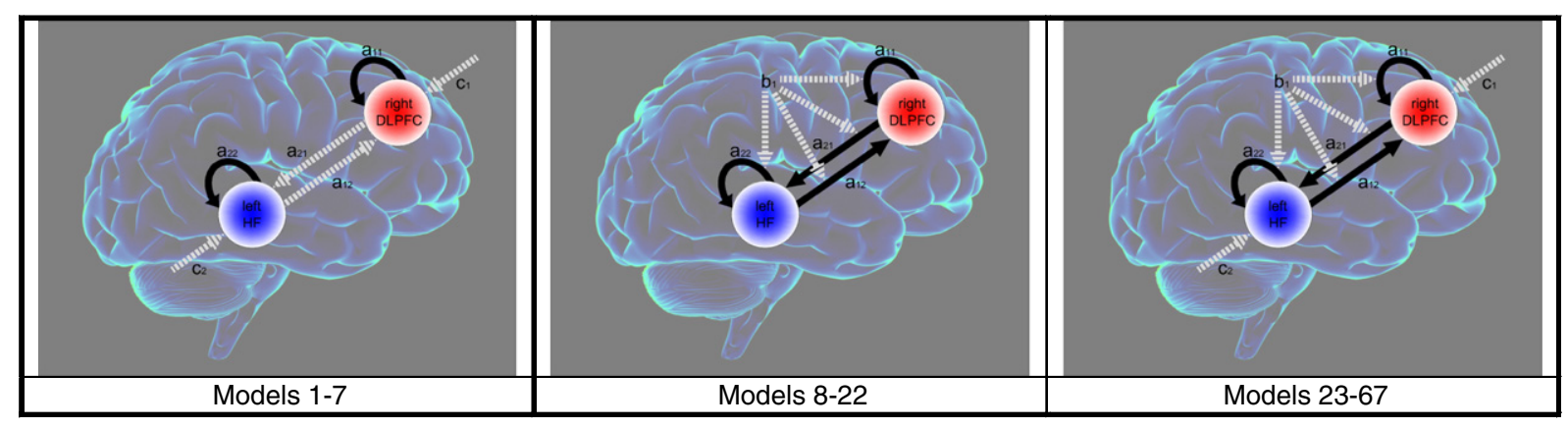

Fig. 1. Space-state model. 


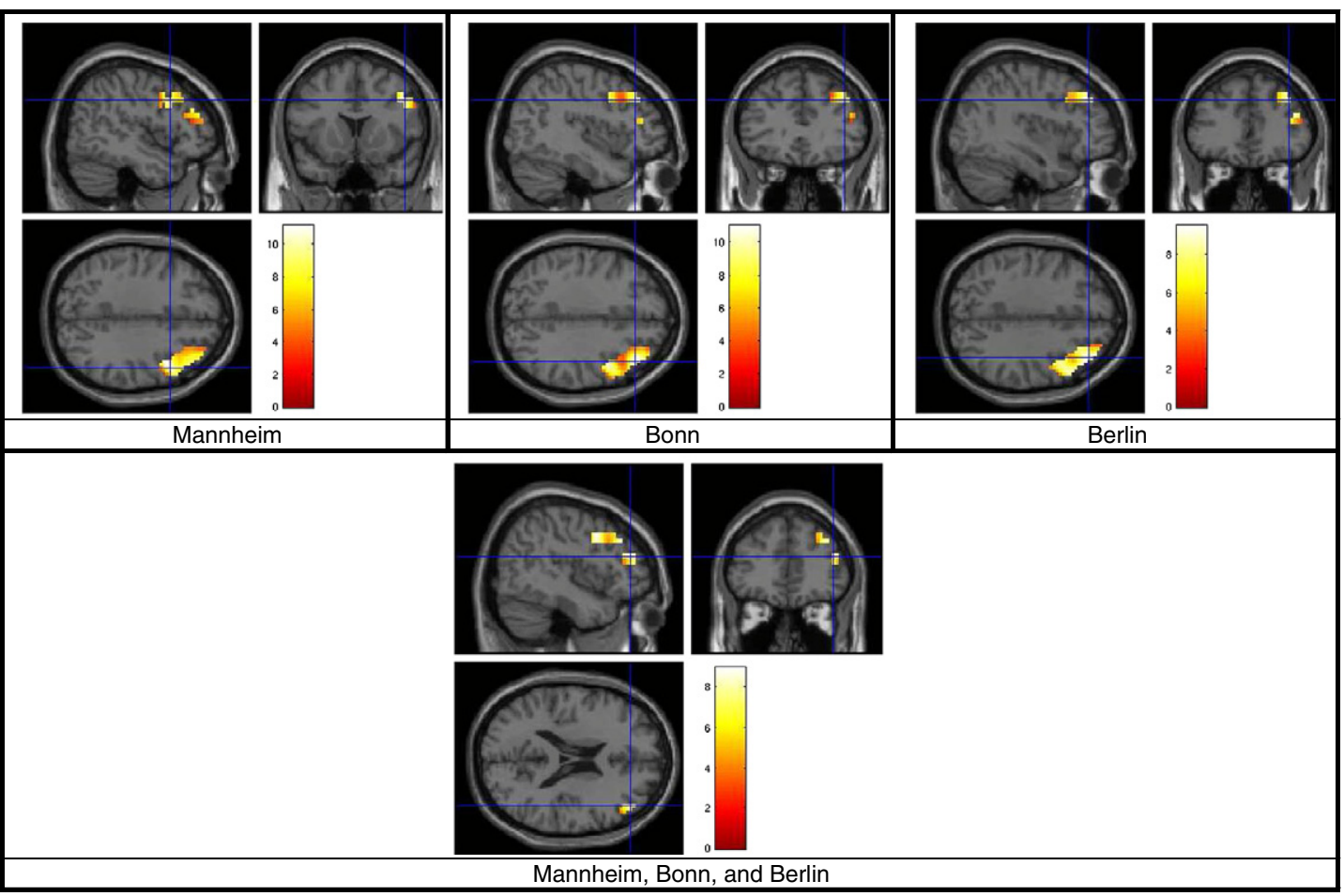

Fig. 2. Activation maps for each location and conjunction map in the right DLPFC.

$\mathrm{HF}(x, y, z=-27,-31,-10 ; \mathrm{T}=8.99 ; \mathrm{p}=0.05$, FWE-corrected $)$ across all three sites.

As described in the Methods section, these conjunction results were subsequently used as group-level functional ROIs to guide the subsequent time series extraction in the right DLPFC and left HF of each individual subject.
$B M S$

We used random effects BMS to determine, from our model space of sixty-seven alternative sDCMs (Fig. 1), the model that provided the best balance between accuracy and complexity for explaining the measured data. The results were fully consistent across the three sites,

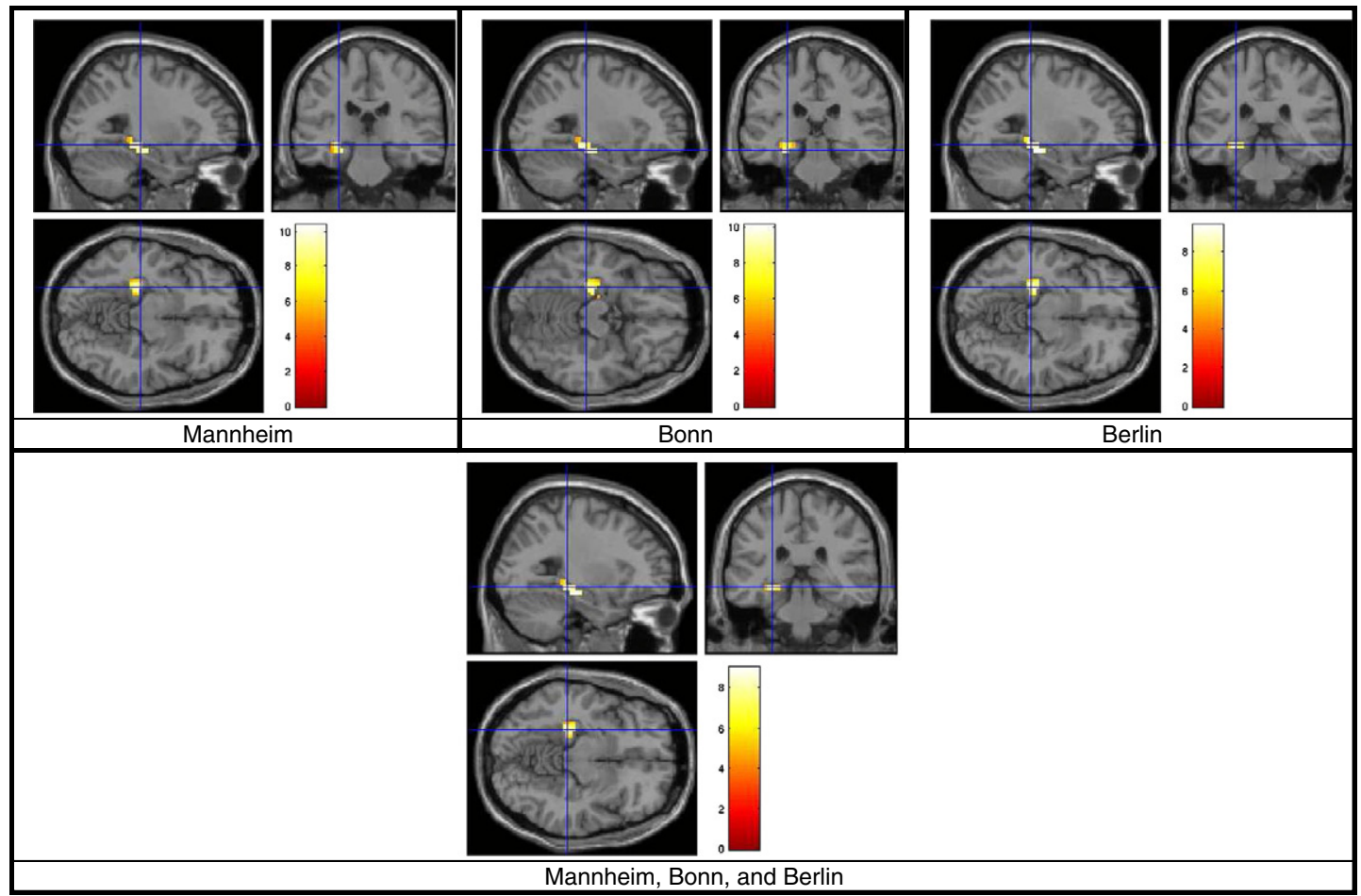

Fig. 3. Deactivation maps for each location and conjunction map in the left HF. 


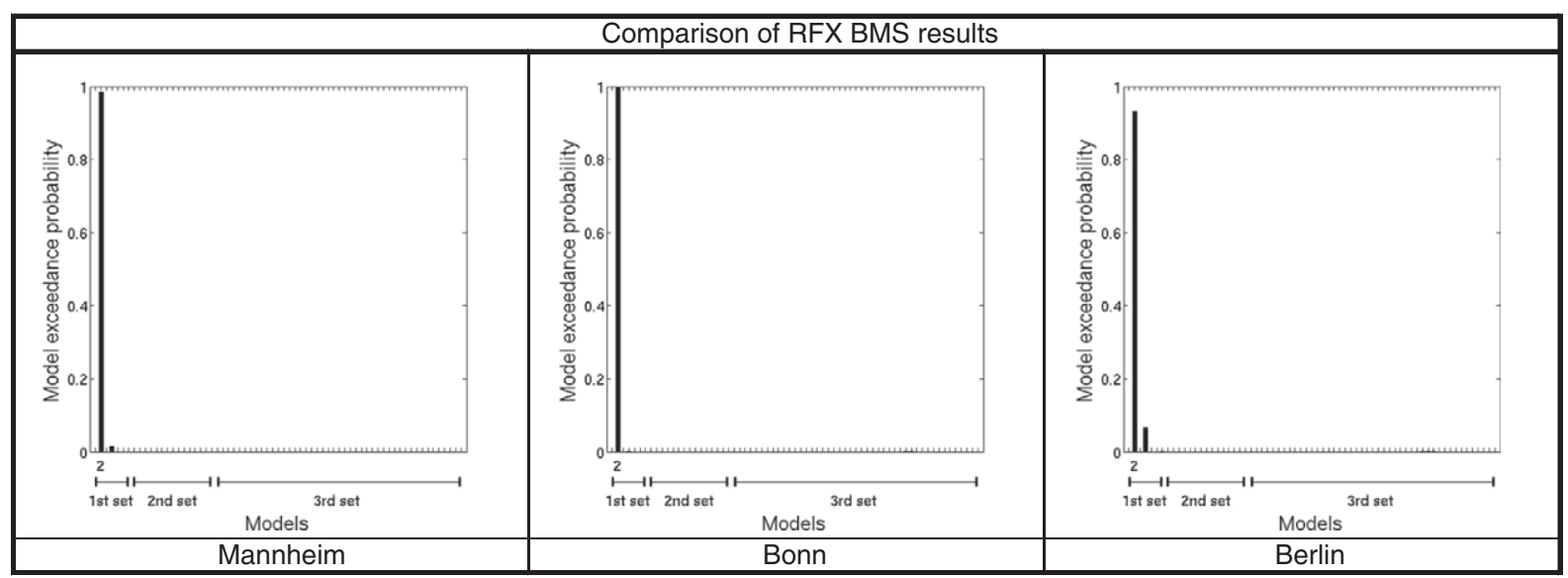

Fig. 4. Comparison of RFX BMS results across the three locations.

revealing the same winning model (model 2; Fig. 1) in Mannheim, Bonn, and Berlin. This model includes a driving influence of the 2-Back condition on both DLPFC and HF, and a unidirectional influence from DLPFC to HF.

Fig. 4 shows the results of BMS Random Effects (RFX) for each location. The exceedance probabilities of model 2 (i.e., the probability that this model is a more likely model than any other model considered) were 0.97 (for the Mannheim cohort), 1.00 (for the Bonn cohort), and 0.93 (for the Berlin cohort), respectively.

\section{sDCM parameter estimates}

As described above, BMS revealed the same winning model in Mannheim, Bonn, and Berlin. For this model, Fig. 5 shows the mean sDCM parameter estimates across participants from each site and the grand average across all sites.

As a further test of consistency of sDCM results across the three sites, we examined potential differences in the parameter estimates of the winning model by performing one-way ANOVA or a Kruskal-Wallis test depending on the distribution of the SDCM parameter estimates previously assessed by a Lilliefors test, see Fig. 6 . Even without correction for multiple comparisons, none of the five parameters contained by model 2 were statistically different across sites.

Clearly we cannot infer that there are no differences between the parameter estimates among the sites (because this would rest upon accepting the null hypothesis). However, we can use a two-way analysis of variance and check if we have sufficient power to detect differences among connections. If this is the case and we are, at the same time, unable to find a significant site by connection interaction, then this absent interaction effect cannot be explained trivially by an underpowered analysis. In other words, we tested whether our analyses were sufficiently sensitive to detect departures from the null hypothesis of differences in connection strengths and yet failed to show any differences between sites.

Based on this rationale, we performed two-way analyses of variance, with connection strengths (adjusted by subtracting the prior mean

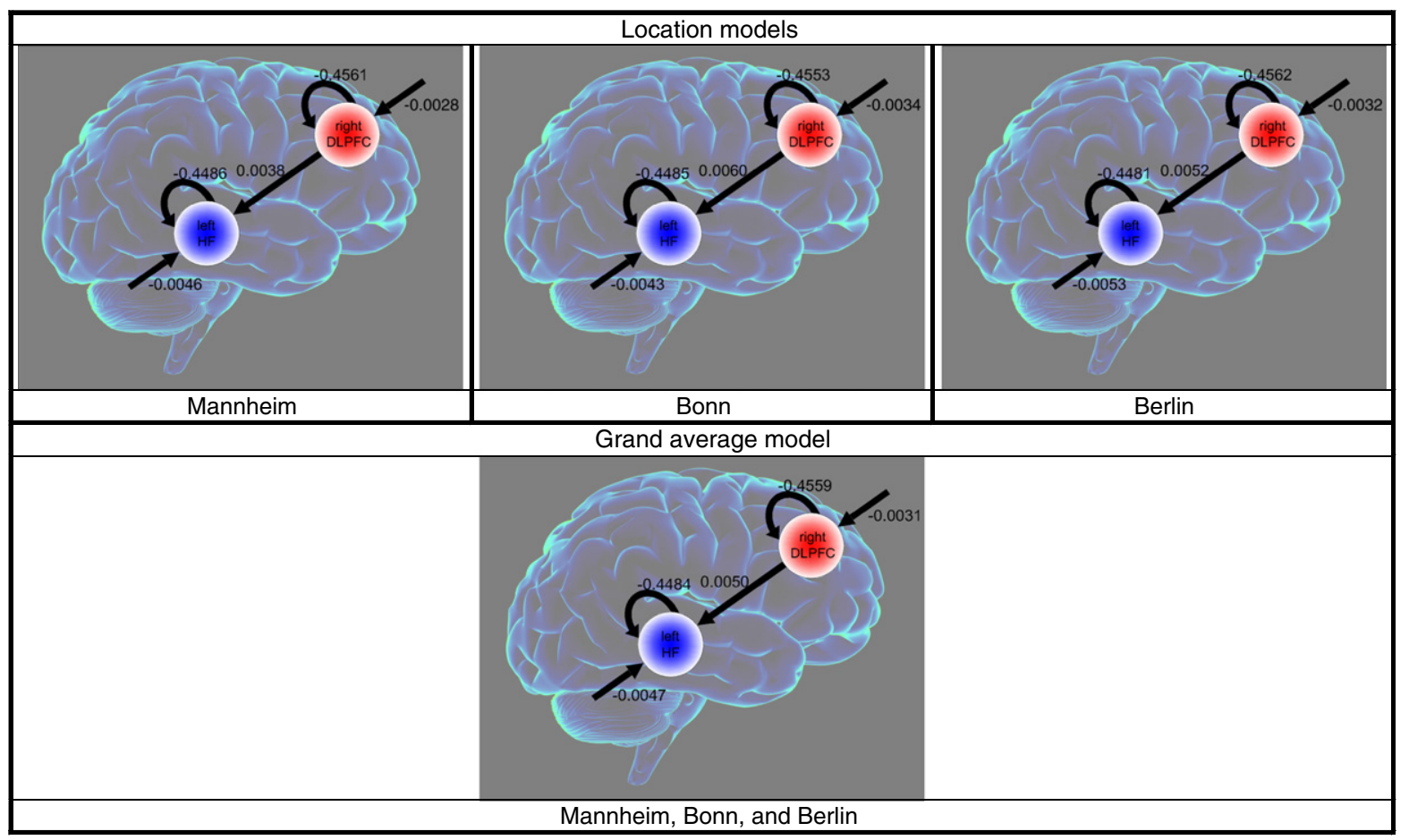

Fig. 5. Mean sDCM parameter estimates across participants from each location and the grand average across all locations. 


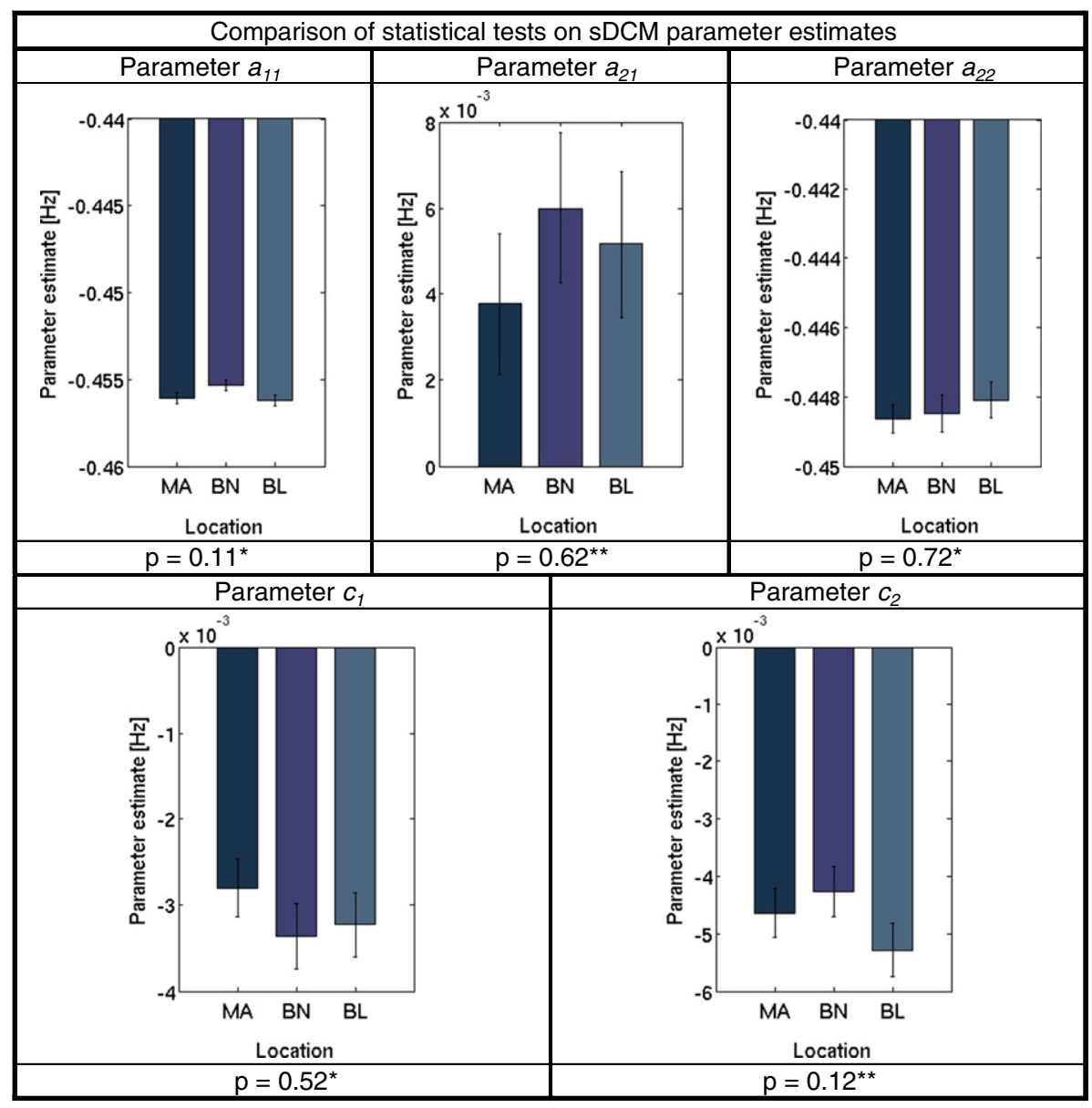

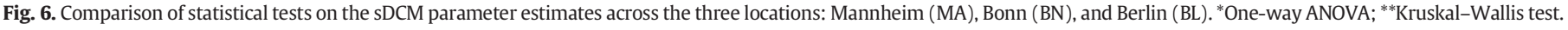

from the posterior mean to avoid trivially significant departures from the null hypothesis of no differences in connection strengths) and sites as the two factors. We found no significant differences between sites using either a non-parametric Friedman-rank test ( $p=0.20$; normality was rejected according to Lilliefors test only in 3 out of 15 repetitions, $\mathrm{p}<0.01$ ) and a two-way ANOVA ( $\mathrm{p}=0.51)$. Similarly, we did not find a significant interaction between sites and model parameter estimates $(p=0.85)$. In contrast, the main effect of connection strength was highly significant $(\mathrm{p}<0.01)$.

\section{Discussion}

In this study, 180 healthy German participants from three different locations were analyzed: Mannheim (60), Bonn (60), and Berlin (60). A conjunction analysis showed that right DLPFC and left HF were consistently activated in participants from all three sites. We then proceeded to constructing a set of sixty-seven sDCMs describing interactions between right DLPFC and left HF during WM. Random effects BMS revealed the same winning model in Mannheim, Bonn, and Berlin. Statistical tests of the sDCM parameter estimates from the winning model revealed no significant difference across the three sites.

In summary, our multi-center study demonstrates a consistent pattern of activation in and connectivity between DLPFC and HF during WM across three different sites. While certainly not an exhaustive test of the robustness of sDCM, this initial reproducibility study speaks favorably to the reliability of sDCM as a method for assessing effective connectivity from fMRI data.

For deterministic DCM, two studies of reliability exist, showing high reproducibility of parameter estimates (Schuyler et al., 2010) and model selection (Rowe et al., 2010) respectively. While reliability tests do not directly address questions of model validity, reliability is an important prerequisite for validity. For deterministic DCM, several studies have been performed that assessed different aspects of its validity. For example, construct validity of deterministic DCM for fMRI has been demonstrated in relation to SEM (Penny et al., 2004b) or large-scale neuronal models (Lee et al., 2006). Predictive validity has been demonstrated in a multimodal rodent study, showing that regional origins of epilepsy spread can be detected (David et al., 2008), and in studies of stroke patients where DCM applied to data from non-lesioned parts of the brain could predict, with nearly perfect accuracy, the absence or presence of a "hidden" lesion, i.e., out of the field of view (Brodersen et al., 2011). Stochastic DCM for fMRI is a more recent development, and so far, only one validation study exists (Daunizeau et al., 2013).

It is notable that in our winning model the maximum a posteriori (MAP) estimates of both our inter-regional connection strengths and driving inputs are rather small (Fig. 5), while visual inspection of our models shows good fit to the data. This implies that the activity in DLPFC and HF can be largely explained by the stochastic innovations (fluctuation terms) that drive activity in DLPFC and HF. This suggests that future refinements of SDCM in SPM should consider the relative precision of priors on stochastic innovations and connectivity parameters.

Nevertheless, the additional (small) explanatory contribution of inter-regional connectivity is very stable across subjects, as demonstrated by our analyses (Fig. 6). Furthermore, removing the influence of task as driving input (models 8-22) or disallowing for the endogenous connection from DLPFC to HF (in models 1-7) clearly deteriorated model goodness, as shown by the results of our BMS analyses. This means that despite their small MAP values, both driving inputs and inter-regional connections play a sufficiently important role in 
explaining regional activity (in DLPFC and HF) that the respective models can be clearly distinguished in terms of their model evidence.

Another interesting (and unexpected) aspect of the quantitative results was that the parameters that scale experimental input (2-Back) were negative for both regions-despite the fact that one region activated and the other deactivated in the SPM. This again speaks to the subtleties of stochastic DCM, in which experimental effects can be modeled by condition-specific fluctuations in hidden neuronal states. In other words, despite the fact that the cognitive set associated with task performance appears to produce an inhibitory afferent drive to both regions, the estimated changes in neuronal activity must more than compensate in the prefrontal region showing an activation (which drives the hippocampal region showing a deactivation). In future work, it may be interesting to test this conjecture using the estimates of hidden neuronal activity in the two regions directly as the basis for statistical analyses. Our hypothesis here would be that the estimated activity in the prefrontal region would be higher, on average, during the 2-Back working memory condition and the converse for the hippocampal region.

It may be helpful to remember that the lumped neuronal activity modeled by DCM for fMRI does not correspond to evoked responses in electrophysiology; rather, it probably reflects very slow fluctuations in the power of high frequency dynamics-that have much slower rate constants than the underlying neuronal activity itself.

Our initial reliability study of sDCM provides a basis for forthcoming clinical and imaging genetics studies. After demonstrating that sDCM provides reliable estimates of prefrontal-hippocampal interactions, we will use this modeling approach in future studies to identify connectivity alterations in genetic risk carriers and patients. Specifically, we will investigate relations between SDCM parameter estimates, behavior, and genetic risk variants that have been implicated in WM and prefrontal-hippocampal connectivity, in healthy volunteers and schizophrenia patients.

\section{Acknowledgments}

This work was funded by grants from the German Ministry for Education and Research through the Bernstein Center for Computational Neuroscience (BMBF, 01GQ1003B) initiative and through the National Genome Research Network (NGFN plus MOODS). K.E. Stephan acknowledges support by the René and Susanne Braginsky Foundation.

\section{Disclosure Statement}

The authors declare that there is no conflict of interest to disclose.

\section{References}

Ashburner, J., Friston, K.J., 2005. Unified segmentation. Neurolmage 26, 839-851. Baddeley, A., 1992. Working memory. Science 255, 556-559.

Barch, D.M., 2005. The cognitive neuroscience of schizophrenia. Annu. Rev. Clin. Psychol.1, 321-353.

Benetti, S., Mechelli, A., Picchioni, M., Broome, M., Williams, S., McGuire, P., 2009. Functional integration between the posterior hippocampus and prefrontal cortex is impaired in both first episode schizophrenia and the at risk mental state. Brain 132, 2426-2436.

Biswal, B., Yetkin, F.Z., Haughton, V.M., Hyde, J.S., 1995. Functional connectivity in the motor cortex of resting human brain using echo-planar MRI. Magn. Reson. Med. $34,537-541$

Brodersen, K.H., Schofield, T.M., Leff, A.P., Ong, C.S., Lomakina, E.I., Buhmann, J.M., Stephan, K.E., 2011. Generative embedding for model-based classification of fMRI data. PLoS Comput. Biol. 7, e1002079.

Callicott, J.H., Mattay, V.S., Bertolino, A., Finn, K., Coppola, R., Frank, J.A., Goldberg, T.E., Weinberger, D.R., 1999. Physiological characteristics of capacity constraints in working memory as revealed by functional MRI. Cereb. Cortex 9, 20-26.

Crossley, N.A., Mechelli, A., Fusar-Poli, P., Broome, M.R., Matthiasson, P., Johns, L.C., Bramon, E., Valmaggia, L., Williams, S.C., McGuire, P.K., 2009. Superior temporal lobe dysfunction and frontotemporal dysconnectivity in subjects at risk of psychosis and in first-episode psychosis. Hum. Brain Mapp. 30, 4129-4137.

Daunizeau, J., Friston, K.J., Kiebel, S.J., 2009. Variational Bayesian identification and prediction of stochastic nonlinear dynamic causal models. Physica D 238, 2089-2118.

Daunizeau, J., Lemieux, L., Vaudano, A.E., Friston, K.J., Stephan, K.E., 2013. An electrophysiological validation of stochastic DCM for fMRI. Front. Comput. Neurosci. 6.
Daunizeau, J., Stephan, K.E., Friston, K.J., 2012. Stochastic dynamic causal modelling of fMRI data: should we care about neural noise? NeuroImage 62, 464-481.

David, O., Guillemain, I., Saillet, S., Reyt, S., Deransart, C., Segebarth, C., Depaulis, A., 2008. Identifying neural drivers with functional MRI: an electrophysiological validation. PLoS Biol. 6, 2683-2697.

Deserno, L., Sterzer, P., Wustenberg, T., Heinz, A., Schlagenhauf, F., 2012. Reduced prefrontal-parietal effective connectivity and working memory deficits in schizophrenia. J. Neurosci. 32, 12-20.

Esslinger, C., Walter, H., Kirsch, P., Erk, S., Schnell, K., Arnold, C., Haddad, L., Mier, D. Opitz von Boberfeld, C., Raab, K., Witt, S.H., Rietschel, M., Cichon, S., MeyerLindenberg, A., 2009. Neural mechanisms of a genome-wide supported psychosis variant. Science 324, 605.

Fletcher, P., 1998. The missing link: a failure of fronto-hippocampal integration in schizophrenia. Nat. Neurosci. 1, 266-267.

Forbes, N.F., Carrick, L.A., McIntosh, A.M., Lawrie, S.M., 2009. Working memory in schizophrenia: a meta-analysis. Psychol. Med. 39, 889-905.

Fornito, A., Zalesky, A., Pantelis, C., Bullmore, E.T., 2012. Schizophrenia, neuroimaging and connectomics. NeuroImage 62, 2296-2314.

Friston, K., Mattout, J., Trujillo-Barreto, N., Ashburner, J., Penny, W., 2007. Variational free energy and the Laplace approximation. Neurolmage 34, 220-234.

Friston, K., Stephan, K.E., Li, B., Daunizeau, J., 2013. Generalised Filtering. Math. Probl. Eng. (in press, Article ID 621670).

Friston, K.J., Frith, C.D., 1995. Schizophrenia: a disconnection syndrome? Clin. Neurosci. 3, 89-97.

Friston, K.J., Harrison, L., Penny, W., 2003. Dynamic causal modelling. Neurolmage 19, $1273-1302$.

Friston, K.J., Li, B., Daunizeau, J., Stephan, K.E., 2011. Network discovery with DCM NeuroImage 56, 1202-1221.

Friston, K.J., Liddle, P.F., Frith, C.D., Hirsch, S.R., Frackowiak, R.S., 1992. The left medial temporal region and schizophrenia. A PET study. Brain 115 (Pt 2), 367-382.

Friston, K.J., Trujillo-Barreto, N., Daunizeau, J., 2008. DEM: a variational treatment of dynamic systems. Neurolmage $41,849-885$

Gazzaley, A., Rissman, J., D'Esposito, M., 2004. Functional connectivity during working memory maintenance. Cogn. Affect. Behav. Neurosci. 4, 580-599.

Goldman-Rakic, P.S., 1994. Working memory dysfunction in schizophrenia. J. Neuropsychiatry Clin. Neurosci. 6, 348-357.

He, H., Sui, J., Yu, Q., Turner, J.A., Ho, B.C., Sponheim, S.R., Manoach, D.S., Clark, V.P., Calhoun, V.D., 2012. Altered small-world brain networks in schizophrenia patients during working memory performance. PLoS One 7, e38195.

Kruger, G., Glover, G.H., 2001. Physiological noise in oxygenation-sensitive magnetic resonance imaging. Magn. Reson. Med. 46, 631-637.

Lee, J., Park, S., 2005. Working memory impairments in schizophrenia: a meta-analysis J. Abnorm. Psychol. 114, 599-611.

Lee, L., Friston, K., Horwitz, B., 2006. Large-scale neural models and dynamic causal modelling. NeuroImage 30, 1243-1254.

Li, B., Daunizeau, J., Stephan, K.E., Penny, W., Hu, D., Friston, K., 2011. Generalised filtering and stochastic DCM for fMRI. Neurolmage 58, 442-457.

Manoach, D.S., Press, D.Z., Thangaraj, V., Searl, M.M., Goff, D.C., Halpern, E., Saper, C.B. Warach, S., 1999. Schizophrenic subjects activate dorsolateral prefrontal cortex during a working memory task, as measured by fMRI. Biol. Psychiatry 45 1128-1137.

Meyer-Lindenberg, A.S., Olsen, R.K., Kohn, P.D., Brown, T., Egan, M.F., Weinberger, D.R. Berman, K.F., 2005. Regionally specific disturbance of dorsolateral prefrontalhippocampal functional connectivity in schizophrenia. Arch. Gen. Psychiatry 62, 379-386.

Nichols, T., Brett, M., Andersson, J., Wager, T., Poline, J.B., 2005. Valid conjunction inference with the minimum statistic. NeuroImage 25, 653-660.

Owen, A.M., McMillan, K.M., Laird, A.R., Bullmore, E., 2005. N-back working memory paradigm: a meta-analysis of normative functional neuroimaging studies. Hum. Brain Mapp. 25, 46-59.

Park, S., Holzman, P.S., 1992. Schizophrenics show spatial working memory deficits Arch. Gen. Psychiatry 49, 975-982.

Paulus, F.M., Krach, S., Bedenbender, J., Pyka, M., Sommer, J., Krug, A., Knake, S., Nothen, M.M., Witt, S.H., Rietschel, M., Kircher, T., Jansen, A., 2013. Partial support for ZNF804A genotype-dependent alterations in prefrontal connectivity. Hum. Brain Mapp. 34, 304-313.

Penny, W.D., Stephan, K.E., Daunizeau, J., Rosa, M.J., Friston, K.J., Schofield, T.M., Leff, A.P., 2010. Comparing families of dynamic causal models. PLoS Comput. Biol. 6, e1000709.

Penny, W.D., Stephan, K.E., Mechelli, A., Friston, K.J., 2004a. Comparing dynamic causal models. Neurolmage 22, 1157-1172.

Penny, W.D., Stephan, K.E., Mechelli, A., Friston, K.J., 2004b. Modelling functional integration: a comparison of structural equation and dynamic causal models. NeuroImage 23 (Suppl. 1), S264-S274.

Pitt, M.A., Myung, I.J., 2002. When a good fit can be bad. Trends Cogn. Sci. 6, 421-425.

Rasetti, R., Sambataro, F., Chen, Q., Callicott, J.H., Mattay, V.S., Weinberger, D.R. 2011. Altered cortical network dynamics: a potential intermediate phenotype for schizophrenia and association with ZNF804A. Arch. Gen. Psychiatry 68 1207-1217.

Riera, J.J., Watanabe, J., Kazuki, I., Naoki, M., Aubert, E., Ozaki, T., Kawashima, R., 2004 A state-space model of the hemodynamic approach: nonlinear filtering of BOLD signals. NeuroImage 21, 547-567.

Rowe, J.B., Hughes, L.E., Barker, R.A., Owen, A.M., 2010. Dynamic causal modelling of effective connectivity from fMRI: are results reproducible and sensitive to Parkinson's disease and its treatment? Neurolmage 52, 1015-1026.

Schlosser, R., Gesierich, T., Kaufmann, B., Vucurevic, G., Hunsche, S., Gawehn, J., Stoeter, P., 2003. Altered effective connectivity during working memory performance in 
schizophrenia: a study with fMRI and structural equation modeling. Neurolmage 19, 751-763.

Schuyler, B., Ollinger, J.M., Oakes, T.R., Johnstone, T., Davidson, R.J., 2010. Dynamic Causal Modeling applied to fMRI data shows high reliability. NeuroImage 49, 603-611.

Sigurdsson, T., Stark, K.L., Karayiorgou, M., Gogos, J.A., Gordon, J.A., 2010. Impaired hippocampal-prefrontal synchrony in a genetic mouse model of schizophrenia. Nature 464, 763-767.

Silver, H., Feldman, P., Bilker, W., Gur, R.C., 2003. Working memory deficit as a core neuropsychological dysfunction in schizophrenia. Am. J. Psychiatry 160, 1809-1816.

Stephan, K.E., Friston, K.J., Frith, C.D., 2009a. Dysconnection in schizophrenia: from abnormal synaptic plasticity to failures of self-monitoring. Schizophr. Bull. 35, 509-527.

Stephan, K.E., Penny, W.D., Daunizeau, J., Moran, R.J., Friston, K.J., 2009b. Bayesian model selection for group studies. Neurolmage 46, 1004-1017.

Stephan, K.E., Penny, W.D., Moran, R.J., den Ouden, H.E., Daunizeau, J., Friston, K.J., 2010. Ten simple rules for dynamic causal modeling. NeuroImage 49, 3099-3109.
Stephan, K.E., Weiskopf, N., Drysdale, P.M., Robinson, P.A., Friston, K.J., 2007. Comparing hemodynamic models with DCM. NeuroImage 38, 387-401.

Weinberger, D.R., Berman, K.F., Suddath, R., Torrey, E.F., 1992. Evidence of dysfunction of a prefrontal-limbic network in schizophrenia: a magnetic resonance imaging and regional cerebral blood flow study of discordant monozygotic twins. Am. J. Psychiatry 149, 890-897.

Wolf, R.C., Vasic, N., Sambataro, F., Hose, A., Frasch, K., Schmid, M., Walter, H., 2009. Temporally anticorrelated brain networks during working memory performance reveal aberrant prefrontal and hippocampal connectivity in patients with schizophrenia. Prog. Neuropsychopharmacol. Biol. Psychiatry 33, 1464-1473.

Zhou, Y., Liang, M., Jiang, T., Tian, L., Liu, Y., Liu, Z., Liu, H., Kuang, F., 2007. Functional dysconnectivity of the dorsolateral prefrontal cortex in first-episode schizophrenia using resting-state fMRI. Neurosci. Lett. 417, 297-302.

Zhou, Y., Shu, N., Liu, Y., Song, M., Hao, Y., Liu, H., Yu, C., Liu, Z., Jiang, T., 2008. Altered resting-state functional connectivity and anatomical connectivity of hippocampus in schizophrenia. Schizophr. Res. 100, 120-132. 